\title{
3 Fakten zur Finanzkrise 2008 und der ihr folgenden Wirtschaftskrise, bezogen auf Wirtschafts- und soziale Daten
}

In diesem Kapitel sollen zunächst überblicksartig jene Faktoren beschrieben werden, die zur Finanzkrise 2008 und im Anschluss daran zu einem massiven Einbruch der Weltwirtschaft führten, sodass einzelne Menschen und ganze Kollektive davon betroffen waren. Im Anschluss wird auf die Situation in Österreich genauer eingegangen.

Die globale Vernetzung des Finanzbereiches ist mit dafür verantwortlich, dass die von den USA ausgehende Krise als die größte seit der großen Depression in den 30er-Jahren des 20. Jahrhunderts beschrieben wird. ${ }^{17}$

Die Finanzkrise nahm 2007 mit dem Platzen der Immobilienblase (Subprime-Krise) in den USA ihren Anfang. Heute geht man davon aus, dass diese verschiedene Ursachen hatte. Als eine Ursache wird eine bestimmte Denkrichtung in den Wirtschaftswissenschaften angesehen, nämlich der sogenannte „Marktfundamentalismus“. Dessen Vertreter meinen, dass „freie Märkte von selbst wirtschaftlichen Wohlstand und Wirtschaftswachstum hervorbringen [...]" (Stiglitz, 2011, S. 11). Ausdruck findet diese Denkrichtung in einer durch die Politik betriebenen Deregulierung des Finanzsektors, die es möglich machte, unkontrolliert Darlehen an Menschen zu vergeben, die eine geringe Bonität und in den meisten Fällen ein geringes Wissen über mögliche Komplikationen und deren Folgen, wie steigende Zinssätze, fehlende Ausfallsversicherungen und Haftungen, hatten. Aus der Anspielung auf diese Klientel stammt der Begriff „Subprime“. Der Besitz eines Eigenheimes hat in den USA auch eine sozialpolitische Dimension. „Miete gilt als Zeichen von geringem sozialen Status und fehlender Solidität"“18 (Hetzer, 2011, S. 143).

Der Abschluss solcher Subprime-Verträge, die aufgrund der geringen Bonität der Klienten mit höheren Zinssätzen verbunden waren, brachte den Kredit-

17 Vergleiche dazu Hetzer (2011, S. 15), Martin-Carrasco et al. (2016, S. 90), Nachtwey (2017, S. 65) oder Stiglitz (2011, S. 33).

18 „Der US-Kongress hatte schon 1977 den „Community Reinvestment Act“ verabschiedet, durch den Banken angehalten wurden, verstärkt Bauprojekte in ärmeren Wohngegenden zu finanzieren [...] Die Regierung unter Bill Clinton verstärkte die Bemühungen, auch Bürgern mit geringem Einkommen sowie Minderheiten den Weg zum eigenen Haus zu ebnen. Die Regierung unter George W. Bush setzte diese Anstrengungen fort" (Hetzer, 2011, S. 143). 
vermittlern hohe Prämien ein und befeuerte so zusätzlich die unlauteren Vergabepraktiken. Der Wohnungs- und Häusermarkt boomte. ${ }^{19}$ Immer mehr Menschen investierten in Immobilien, was letztlich zu einer Blase und einem enormen Wertverlust der Objekte führte, als sie platzte. Da die Kreditschulden der Eigentümer den Wert des Eigentums oft vielfach überstiegen, verloren die Menschen nicht nur ihre Häuser, sondern blieben auch auf ihren Schulden sitzen.

Die Kredite mit geringer Bonität wurden von den Finanzinstituten mit anderen zu neuen Finanzprodukten, sogenannten Derivaten ${ }^{20}$, kombiniert, „verbrieft“ und weiter verkauft (vgl. Hetzer, 2011, S. 8). Diese Finanzprodukte ,[...] erlaubten es den Banken, einen Großteil ihrer Problemkredite zu kaschieren, sie nicht in ihren Bilanzen auszuweisen und so ihren tatsächlichen Verschuldungsgrad zu erhöhen [...]“ (Stiglitz, 2011, S. 17). Ein Anreizsystem, das den Bankmanagern hohe Bonuszahlungen versprach, förderte diesen riskanten Handel.

Wie sich später zeigen sollte, investierten etwa Pensionsfonds oder Versicherungen in solche Produkte; deren Kunden verloren aufgrund der Täuschung in der Folge jegliche Absicherung. Die Spekulationswelle hatte weite Teile des Finanzsektors erfasst.

Die ursprüngliche Intention dieser Finanzinstrumente wäre gewesen, das Risiko unter den Teilnehmern des globalen Finanzmarkts zu verteilen (vgl. Hetzer, 2011, S. 145). Am Beispiel der CDS (Credit Default Swaps), einer Art Kreditversicherung, die unabhängig vom Verhältnis zwischen Schuldner und Gläubiger gehandelt wurden, kann man nachvollziehen, wie sie zu einem Spekulationsinstrument wurden. ${ }^{21}$ Durch das einfließende Fremdkapital kommt eine Art Hebelwirkung zustande, die sowohl hohe Gewinne als auch Verluste bei niedrigen Anfangsinvestitionen ermöglicht (vgl. Hetzer, 2011, S. 146). Schuldtitel wurden mehrfach versichert, sodass am Höhepunkt der Spekulationswelle das Volumen der CDS enorme Ausmaße erreicht hatte.

19 „In den Jahren vor der Krise entfielen 30 bis 40 Prozent aller Investitionen auf den Bausektor [...]“" (Stiglitz, 2011, S. 382).

20 „Bislang wurden nur die Einkünfte aus Hypothekenzahlungen in ,Wertpapiere 'verpackt und gehandelt. Später kam die bemerkenswerte Idee, die mit diesen Wertpapieren verbundenen Risiken in Gestalt von Kreditderivaten (Credit Default Swaps (CDSs)), das heißt Kreditausfallsversicherungen, oder Collaterized Debt Obligations (CDOs), also in Fonds gebündelte und verbriefte Kredite unterschiedlicher Qualität, zu verkaufen“ (Hetzer, 2011, S. 144).

21 „Sie [US-Banken und Finanzinstitute] hatten neue Produkte entwickelt, die sie als Instrumente zur Risikoabsicherung anpriesen, während sie in Wirklichkeit so gefährlich waren, dass sie die Stabilität des US-Finanzsystems gefährdeten" (Stiglitz, 2011, S. 33). 
"The use of credit default swaps has become increasingly popular over time. Between 2002 and 2007, gross notional amounts outstanding grew from below USD 2 trillion to nearly USD 60 trillion. By the end of 2007, the outstanding amount was $\$ 62.2$ trillion, falling to $\$ 38.6$ trillion by the end of 2008." (Angelini, 2012, S. 584)

Die Banken arbeiteten mit Rating-Agenturen zusammen, die Wertpapiere, aber auch bereits marode Banken mit Triple $\mathrm{A}$, der höchsten Bonitätsnote, bewerteten und dafür hohe Zahlungen kassierten. Dieses risikogeleitete Handeln, kombiniert mit unlauteren Geschäftspraktiken, führte dazu, dass das Vertrauen in den Bankensektor verloren ging. Als die Zinsen stiegen, erwiesen sich die Kredite als ungesichert und die Blase platzte. Hunderttausende Menschen verloren ihre Häuser, der Immobilienmarkt brach zusammen. ${ }^{22}$ Banken kamen in enorme Schwierigkeiten.

2008 erreichte die Bankenkrise mit dem Bankrott der Investmentbank Lehman Brothers einen ersten Höhepunkt und dehnte sich aufgrund der globalen Vernetzung des Finanzmarktes über den Globus aus. Die Folge war, dass Banken auch in europäischen Staaten zahlungsunfähig wurden. Vielfach musste der Staat eingreifen, um Banken zu retten, was die Verschuldung der betroffenen Staaten entsprechend erhöhte und in weiterer Folge zu Ausgabenkürzungen und/oder Steuererhöhungen führte. ${ }^{23}$ Zugleich war die Vergabe von neuen Krediten durch Banken stark eingeschränkt. Die Industrieproduktion ging zurück und die Weltwirtschaft schlitterte in eine Rezession.

Die Gesamtheit der Folgen führte in Europa zur sogenannten Staatsschuldenkrise, die mit der Herabstufung der Kreditwürdigkeit einzelner europäischer Länder, extremen Sparprogrammen, die besonders die Sozialleistungen betrafen, und erhöhter Arbeitslosigkeit einherging. ${ }^{24}$ Das Bruttoinlandsprodukt schrumpfte und eine Wirtschaftskrise war die Folge (vgl. Bundesministerium für Arbeit, 2017, S. 351). Für Stiglitz war das einzig Überraschende an dieser Krise, dass sie für viele überraschend kam (vgl. Stiglitz, 2011, S. 27). In den Banken selbst änderte sich aufgrund des fehlenden politischen Drucks nur teilweise etwas an den Konditionen für Manager (vgl. Piketty, 2015, S. 64). Ihnen wurden weiter

22 Im Mai 2010 lag die Zahl der Baubeginne bei Einfamilienhäusern um mehr als zwei Drittel unter dem Niveau vom Mai 2005 und um die Hälfte unter dem Niveau fünfzehn Jahre früher, im Mai 1995“ (Stiglitz, 2011, S. 379).

23 Im Vorwort zu Hetzers Buch schreibt Martin Schulz: „[...] laut Internationalem Währungsfonds (IWF) sprachen die Regierungen der USA, Großbritanniens und der Euro-Zone bis April 2009 Garantien im Wert von 8955 Milliarden Dollar aus" (Hetzer, 2011, S. 8).

$24, \ldots[. .$.$] allein in China gingen 20$ Millionen Arbeitsplätze verloren, und viele Millionen Menschen verarmten" (Stiglitz, 2011, S. 9). 
Boni in Millionenhöhe ausbezahlt, was den ganzen Bankensektor in Misskredit brachte (vgl. Stiglitz, 2011, S. 392).

Der Graubereich zwischen Kriminalität, Wirtschaft und Politik, der als eine Ursache für die Krise angesehen wird, spielt auch in den Interviews, die ich geführt habe, eine große Rolle. Diese Entwicklungen trugen auch ihren Teil zur steigenden Politikverdrossenheit bei. ${ }^{25}$

Den zehn größten europäischen Banken gelang es dennoch, wie Piketty schreibt, 2009 ihre Profite auf 50 Milliarden Euro zu steigern, indem „die Zentralbanken den Banken Geld zu sehr niedrigen Zinssätzen geliehen haben - Geld, dass [sic!] diese in der Folge zu höheren Zinssätzen anderen Akteuren leihen konnten: den Haushalten, den Unternehmen und, vor allem, den Staaten“ (Piketty, 2015, S. 63).

Dazu kommt, dass sich dem Finanzsektor, der durch die staatlichen Rettungspakete stabilisiert worden war, durch die Staatsschuldenkrise ein neues Spekulationsfeld eröffnete, weil der Finanzierungsbedarf von Staaten stieg. Es wurde auf den Kursverfall von Staatsanleihen gewettet (Stiglitz, 2011, S. 400). Länder wie etwa Griechenland oder Spanien standen am Abgrund. Was für die Banken galt, sollte nicht für die vom Bankrott bedrohten Länder der europäischen Gemeinschaft gelten. Stiglitz' Prognose im Nachwort zur PaperbackAusgabe seines Buches sollte sich bewahrheiten:

„Europa wird den Krisenländern [...] im äußersten Notfall, in der letzten Minute und mit drückenden Auflagen zur Hilfe kommen. Die auferlegte strenge Haushaltsdisziplin wird in den betroffenen Ländern nicht nur zu sozialer Not führen, sondern die Wirtschaft der Eurozone schwächen und in der Bevölkerung die Unterstützung für die europäische Integration untergraben " (Stiglitz, 2011, S. 407).

Die Kritik an den Institutionen der europäischen Gemeinschaft ist ein wesentlicher Faktor, der zu den Krisenfolgen gehört, worauf im Laufe der Arbeit in den Kapiteln 8.2.5 (Soziale Gerechtigkeit) und 8.2.8 (Quellen der Unzufriedenheit) noch genauer eingegangen wird.

25 „Gewöhnliche Bürger mögen die Komplexität der Makroökonomik nicht verstehen, sie mögen nicht verstehen, auf welche Weise Haushaltskürzungen zu einer Vertiefung der Rezession und einem weiteren Arbeitsplatzabbau führen, aber sie verstehen voll und ganz, dass das größte Rettungspaket aller Zeiten den Banken zugute kam“ (Stiglitz, 2011, S. 394). 


\subsection{Krisenfolgen für Österreich}

Im folgenden Abschnitt sollen die Krisenfolgen für Österreich zusammengefasst werden, weil sie den Hintergrund für die Erfahrungen meiner Interviewpartner bilden. Der Sozialbericht des Ministeriums für Arbeit, Soziales und Konsumentenschutz aus dem Jahr 2017 dient als Basis für die hier zusammengestellten Daten und Fakten.

In Österreich kam es infolge der Finanzkrise zu einem erheblichen Rückgang des Bruttoinlandsprodukts, wie man an Abbildung 1, die die BIP-Veränderung (real) ${ }^{26}$ in Prozent gegenüber dem Vorjahr darstellt, erkennen kann. Die staatlichen Interventionen in Form von zwei Konjunkturpaketen und einer vorgezogenen Steuerreform konnten den Krisenfolgen einigermaßen gegensteuern; „gemessen am Bruttoinlandsprodukt zeigte sich, dass die stabilisierenden Krisenmaßnahmen in Österreich (mit ca. $4 \%$ des BIP) zu den größten im OECDVergleich gehörten“ (Bundesministerium für Arbeit, 2017, S. 347). Einerseits wurde der Bankensektor gestützt ${ }^{27}$, andererseits wurden politische Entscheidungen im Sinne einer Verteilungsgerechtigkeit getroffen.

„Neben der Einführung eines Solidarbeitrags von Personen mit sehr hohen Einkommen wurden auch mit Hilfe der Anhebung der Höchstbeitragsgrundlage und mit der Streichung der Spekulationsfrist bei Immobilienverkäufen diesbezügliche Akzente gesetzt" (Bundesministerium für Arbeit, 2017, S. 353).

Die Zahl der Arbeitslosen stieg infolge der aktiven Arbeitsmarktpolitik und der Wirkung der Konjunkturprogramme wesentlich weniger stark als in fast allen anderen EU-Ländern (siehe dazu Abb. 2); von März 2008 bis März 2009 stieg die Zahl der beim Arbeitsmarktservice gemeldeten Arbeitslosen zwar um

26 Dabei handelt es sich um die inflationsbereinigten Veränderungen.

27 „Der österreichische Bankensektor wurde durch Übernahme von Haftungen sowie Gewährung von Darlehen an Bank- und Finanzinstitute, durch Zuführung von Eigenmitteln und z. T. durch Übernahme von Eigentumsrechten in Ausnahmefällen (z. B. Kommunalkredit, Hypo Alpe Adria) massiv gestützt. Bereits damals wurde vom Gesetzgeber ein finanzieller Rahmen von bis zu 22 Milliarden EUR zur Verfügung gestellt, wobei die Instrumente ,Zuführung von Eigenkapital' sowie ,Übernahme von staatlichen Haftungen' am stärksten in Anspruch genommen wurden. Der österreichische Fiskalrat bezifferte indessen, dass bis Ende 2015 insgesamt 35,7 Mrd EUR der Bruttoverschuldung auf Maßnahmen im Rahmen des Bankenpakets zurückzuführen sind“" (Bundesministerium für Arbeit, 2017, S. 353 f.). 


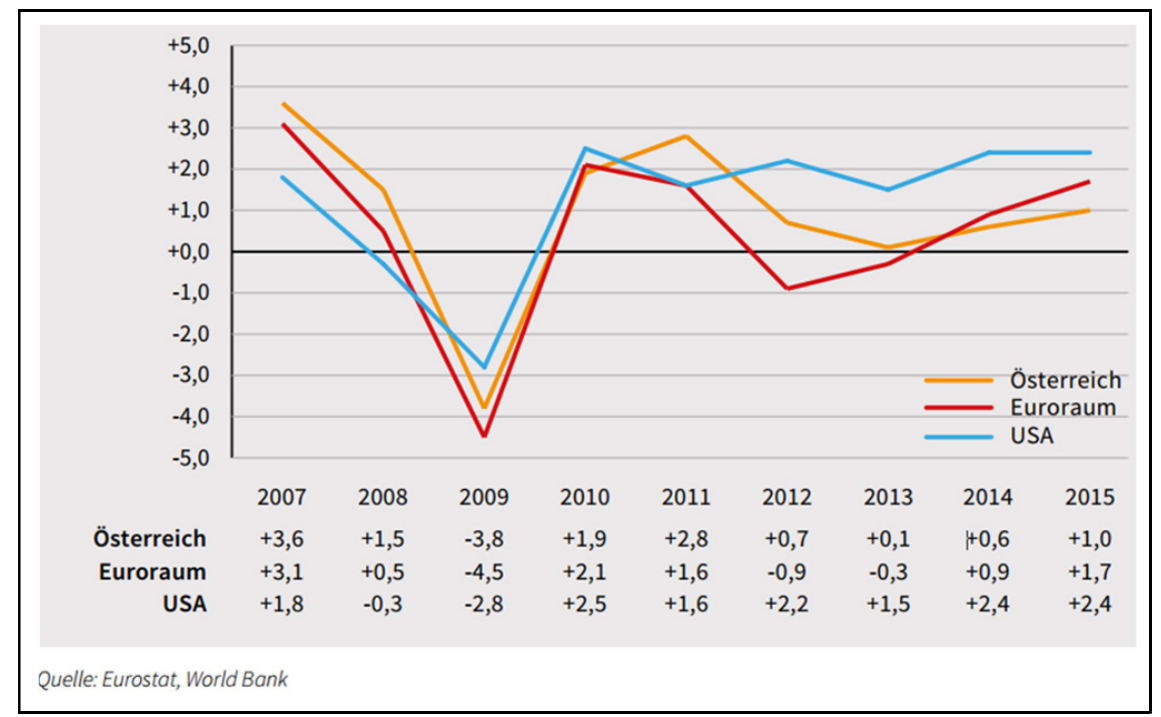

Abbildung 1: Reale BIP-Veränderung gegenüber dem Vorjahr in Prozent (Bundesministerium für Arbeit, 2017, S. 347)

29 Prozent, fiel aber wieder leicht in den zwei folgenden Jahren und lag im zweiten Quartal 2016 bei rund 6 Prozent, was Österreich im Vergleich zu anderen EU-Staaten im unteren Mittelfeld positioniert (vgl. Bundesministerium für Arbeit, 2017, S. 348).

Von Arbeitslosigkeit in einem überdurchschnittlichen Ausmaß bedroht sind vor allem Männer und Personen ab dem Alter von $50^{28}$, Personen mit einem geringem Bildungsgrad ${ }^{29}$ und Ausländer ${ }^{30}$, wenngleich auch die Akademiker-

28 „Zwischen 2008 und 2015 erhöhte sich der Anteil der Männer an den gesamten arbeitslosen Personen um etwa zwei Prozentpunkte auf 58 Prozent" (Bundesministerium für Arbeit, 2017, S. 356).

29 „Personen, die nur über einen Pflichtschulabschluss verfügen, stellen in absoluten Zahlen mit einer Zunahme um fast 54.000 auf rund 141.000 Arbeitslose bis zum Jahr 2015 die größte Risikogruppe dar [...]“ (Bundesministerium für Arbeit, 2017, S. 356).

30 „Lag der Anteil der Personen mit österreichischer Staatsbürgerschaft an den gesamten Arbeitslosen vor Beginn der Krise bei 82 Prozent, so sank dieser Anteil bis 2015 um rund 10 Prozentpunkte auf 73 Prozent“" (Bundesministerium für Arbeit, 2017, S. 356). 


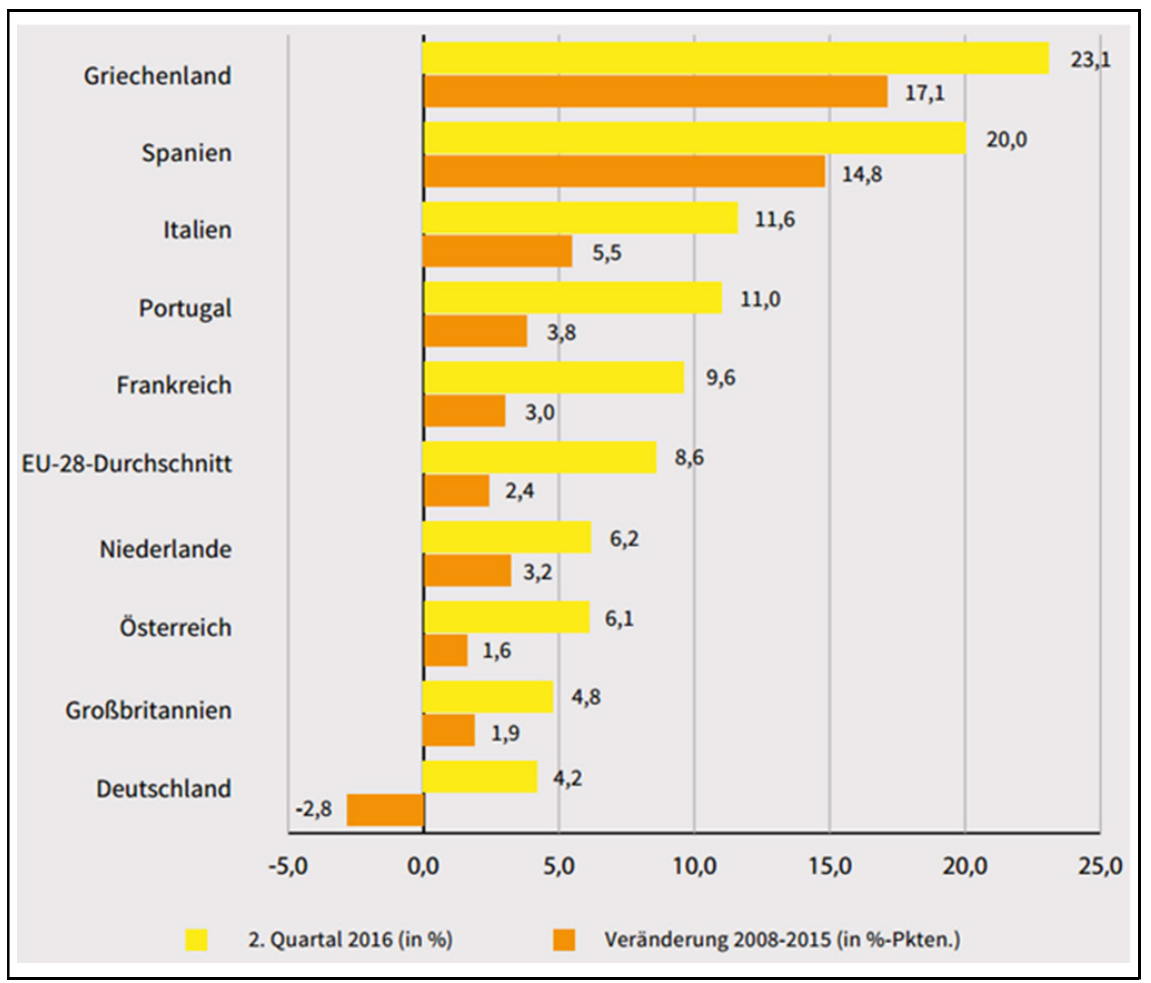

Abbildung 2: Arbeitslosigkeit - Vergleich ausgewählter EU-Mitgliedstaaten seit 2008 und Stand 2016 (Bundesministerium für Arbeit, 2017, S. 349)

arbeitslosigkeit zugenommen hat (vgl. Bundesministerium für Arbeit, 2017, S. 376).

Die fehlende Bindung an den Arbeitgeber im Sinne einer längerfristigen Beschäftigung, die von gegenseitigen Verpflichtungen geleitet wurde und über Jahrzehnte den Status Quo darstellte, hat sich nach der Krise noch einmal verschärft. Es kommt zu einer Veränderung der Beschäftigungsverhältnisse, von der besonders Frauen, schlecht ausgebildete Arbeitnehmer, aber auch vermehrt Akademiker betroffen sind. Die Zahl der Teilzeitverträge steigt. Es zeigt sich auch,

„dass die Zuwächse des unteren Einkommensviertels - aber auch des Medianeinkommens - deutlich unter der Inflation für diesen Zeitraum liegen (lag insgesamt bei 12,2\%), womit hier Reallohnverluste bestehen " (Bundesministerium für Arbeit, 2017, S. 363). 
Tabelle 1: Entwicklung unselbstständig Erwerbstätige 2008-2015, nach Voll-/Teilzeit und Geschlecht (Bundesministerium für Arbeit, 2017, S. 360)

\begin{tabular}{|c|c|c|c|c|c|c|c|}
\hline & \multirow{2}{*}{$\begin{array}{l}\text { Unselbstständig } \\
\text { Erwerbstätige } \\
\text { im Jahr } 2015\end{array}$} & \multicolumn{2}{|c|}{$\begin{array}{l}\text { Gesamtentwicklung } \\
\text { Veränd. seit } 2008\end{array}$} & \multicolumn{2}{|c|}{$\begin{array}{l}\text { Vollzeit-Erwerbstätige } \\
\text { Veränd. seit } 2008\end{array}$} & \multicolumn{2}{|c|}{$\begin{array}{l}\text { Teilzeit-Erwerbstätige } \\
\text { Veränd. seit } 2008\end{array}$} \\
\hline & & absolut & in $\%$ & absolut & in $\%$ & absolut & in $\%$ \\
\hline Insgesamt & 3.609 .200 & +155.900 & $+4,5 \%$ & -57.400 & $-2,2 \%$ & +213.400 & $+26,5 \%$ \\
\hline Männer & 1.875 .900 & +32.100 & $+1,8 \%$ & -28.100 & $-1,6 \%$ & +60.200 & $+49,7 \%$ \\
\hline Frauen & 1.751 .300 & +123.900 & $+7,6 \%$ & -29.300 & $-3,1 \%$ & +153.200 & $+22,4 \%$ \\
\hline
\end{tabular}

Die im internationalen Vergleich relativ niedrigen Arbeitslosenzahlen sind zum Teil aber durch eine zunehmende Tendenz zu Teilzeitbeschäftigungsverhältnissen, insbesondere von Frauen, bedingt. ${ }^{31}$ Auch die Zahl der geringfügig Beschäftigten hat zugenommen.

„Im Jahr 2015 gab es rund 68600 mehr geringfügig Beschäftigte als 2008. Auch diese Entwicklung ist in den Kontext der angespannten Arbeitsmarktlage zu setzen, da der monatliche Richtwert für geringfügige Beschäftigung ${ }^{32}$ als Zuverdienstgrenze beim Arbeitslosengeld und bei der Notstandshilfe sowie auch beim Bezug vorzeitiger Pensionsleistungen [...] herangezogen wird" (Bundesministerium für Arbeit, 2017, S. 361).

In Tabelle 1 sieht man die Veränderung der Zahlen der unselbstständig Erwerbstätigen zwischen 2008 und 2015, aufgegliedert nach Vollzeit, Teilzeit und Geschlecht.

31 „Während die Frauen-Teilzeitquote von 2008 bis 2015 um 5,7 Prozentpunkte angewachsen ist und am Ende dieses Zeitraums 47,8 Prozent betrug, stieg diese bei den Männern im selben Zeitraum um 3,2 Prozentpunkte, erreichte aber auch 2015 den vergleichsweise niedrigen Wert von 9,8 Prozent" (Bundesministerium für Arbeit, 2017, S. 359).

322016 betrug die monatliche Geringfügigkeitsgrenze 415,72 Euro (vgl. Bundesministerium für Arbeit, 2017, S. 361). 


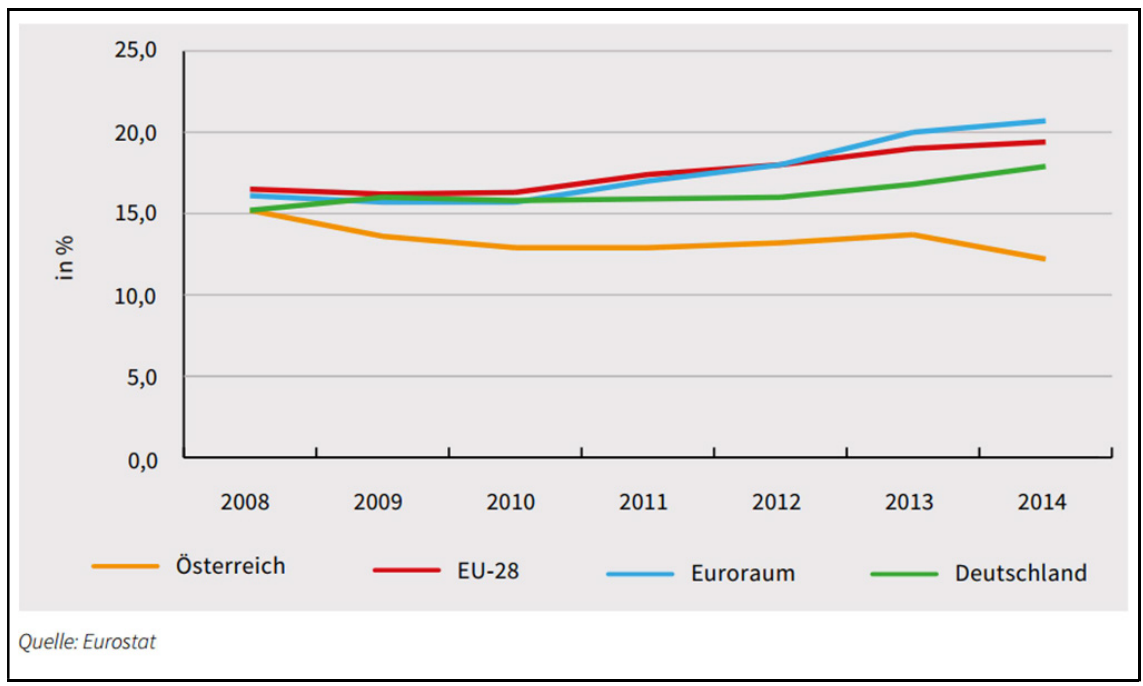

Abbildung 3: Armutsgefährdungsquote 2008-2014 (Bundesministerium für Arbeit, 2017, S. 371)

Die Einführung der bedarfsorientierten Mindestsicherung (BMS) trug dazu bei, dass die Zahl der von Armut gefährdeten Menschen sich im EU-Vergleich trotz Krisenfolgen weiter reduzierte. ${ }^{33}$ In Abbildung 3 wird ersichtlich, wie sich die Armutsgefährdungsquote in Österreich im Vergleich entwickelt hat.

Was die Maßnahmen in Österreich zeigen, ist, dass in wirtschaftlichen Krisenzeiten sogenannte ,automatische Stabilisatoren des Sozialstaats (v. a. Arbeitslosenversicherungsleistungen) über Krisenzeiten armutsvermeidend wirken [... $]^{\text {" }}$ (Bundesministerium für Arbeit, 2017, S. 370), so die Kaufkraft stärken, zu einer Belebung der Wirtschaft führen und den gesellschaftlichen Zusammenhalt festigen.

Aber nicht nur das: existenzbedrohende oder -gefährdende Lebensbedingungen sind auch psychische Destabilatoren, sodass in Krisenzeiten gerade Menschen am unteren Rand der Gesellschaft verstärkt dem Risiko einer Erkrankung

33 „Während 2008 mit einer Armutsgefährdungsquote von 15,2 \% exakt dieselbe Ausgangslage [wie in Deutschland] vorlag, nahm die im Jahr 2008 verankerte Quote in Österreich bis 2014 deutlich ab (12,2\%), während sich jene in Deutschland im selben Zeitraum auf 17,9\% erhöhte. Auch die Durchschnittswerte der EU und des Euroraums weisen steigende verankerte Armutsquoten auf" (Bundesministerium für Arbeit, 2017, S. 371). 


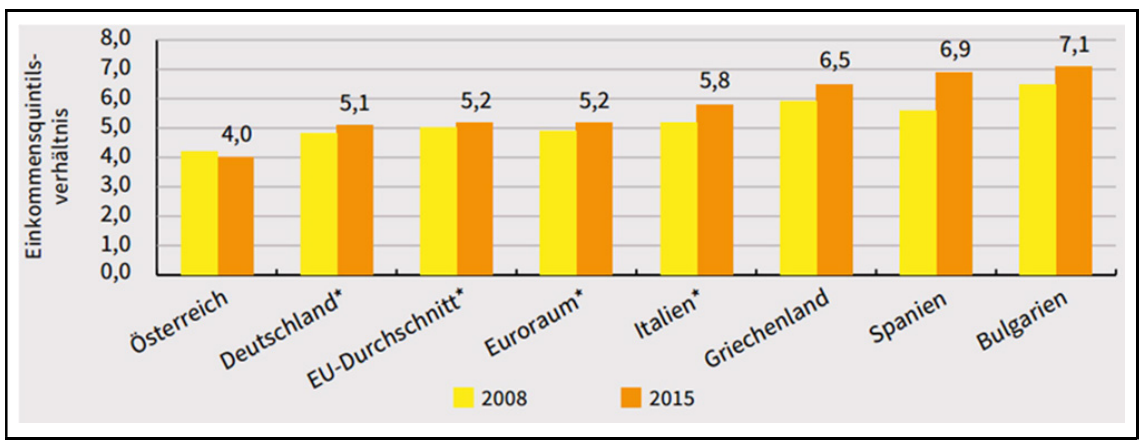

Abbildung 4: Ungleichheit der Einkommensverteilung (Einkommensquintilsverhältnis $^{34}$ ), Vergleich ausgewählter EU-Staaten, 2008-2015 (Bundesministerium für Arbeit, 2017, S. 367)

ausgesetzt sind. Darüber wird in Kapitel 7.1 (Auswirkungen der Krise auf die psychische Gesundheit) mehr zu lesen sein.

Interessant ist, dass in Österreich die Ungleichheit in der Einkommensverteilung nach der Krise nicht zugenommen hat. Allerdings sind dabei ,die Vermögens- und Kapitalerträge [...] nur teilweise erfasst (vor allem hohe Kapitaleinkommen sind untererfasst)“ (Bundesministerium für Arbeit, 2017, S. 368). Im innereuropäischen Vergleich hat die Ungleichheit sehr wohl zugenommen, wie auch aus Abbildung 4 ersichtlich wird.

„So ist zum Beispiel das BIP Deutschlands vom 10,4-Fachen des BIP Griechenlands im Jahr 2007 auf das 15-Fache des griechischen BIP im Jahr 2015 angestiegen. Aber die Spaltung führte auch zu einer Zunahme der Ungleichheit in den Krisenländern" (Stiglitz, 2016, S. 17).

Ebenso in Betracht zu ziehen sind durch Krisenereignisse veränderte politische Einstellungen, optimistische bzw. pessimistische Zukunftserwartungen, die sich auf die Lebensplanung der Menschen auswirken, sowie stabilisierende Faktoren, die Menschen solch tiefgreifende Einschnitte bewältigen lassen. In Tabelle 2 wird eine Erhebung zum subjektiven Wohlbefinden präsentiert.

34 „Darunter ist jener Wert zu verstehen, der das Verhältnis des Gesamteinkommens jener $20 \%$ der Bevölkerung mit dem höchsten Einkommen (oberes Einkommensfünftel od. -quintil) zum Gesamteinkommen jener $20 \%$ mit dem niedrigsten Einkommen (unteres Einkommensfünftel) beschreibt" (Bundesministerium für Arbeit, 2017, S. 367). 
Tabelle 2: Daten zu subjektivem Wohlbefinden und Einstellungen in Österreich (Bundesministerium für Arbeit, 2017, S. 375)

\begin{tabular}{|c|c|c|c|}
\hline Antworten u. Fragestellungen & $\begin{array}{l}\text { Ergebnis } 2006 \\
\text { (vor der Krise) }\end{array}$ & Ergebnis 2014 & $\begin{array}{l}\text { Veränderung in } \\
\text { Prozentpunkten }\end{array}$ \\
\hline Sehr hohe generelle Lebenszufriedenheit & $17,2 \%$ & $15,0 \%$ & $-2,2$ \\
\hline $\begin{array}{l}\text { Frage nach dem Glücksempfinden: } \\
\text { Sehr glücklich }\end{array}$ & $31,9 \%$ & $27,3 \%$ & $-4,6$ \\
\hline Sehr guter subjektiver Gesundheitszustand & $32,3 \%$ & $31,0 \%$ & $-1,3$ \\
\hline $\begin{array}{l}\text { Fühle mich in meiner Wohngegend draußen } \\
\text { abends sehr unsicher }\end{array}$ & $3,6 \%$ & $2,6 \%$ & $-1,0$ \\
\hline $\begin{array}{l}\text { Überhaupt kein Vertrauen in das Europäische } \\
\text { Parlament }\end{array}$ & $12,8 \%$ & $18,8 \%$ & $+6,0$ \\
\hline $\begin{array}{l}\text { Gänzlich kein Vertrauen gegenüber dem } \\
\text { Rechtssystem }\end{array}$ & $2,8 \%$ & $5,5 \%$ & $+2,7$ \\
\hline Kein oder fast kein Vertrauen in Politiker & $25,5 \%$ & $23,8 \%$ & $-1,7$ \\
\hline $\begin{array}{l}\text { Die Europäische Integration ist bereits zu weit } \\
\text { fortgeschritten }\end{array}$ & $11,4 \%$ & $20,4 \%$ & $+9,0$ \\
\hline $\begin{array}{l}\text { Starke Unzufriedenheit mit der heimischen } \\
\text { Wirtschaftssituation }\end{array}$ & $2,5 \%$ & $5,6 \%$ & $+3,1$ \\
\hline $\begin{array}{l}\text { Starke Zustimmung: Regierung sollte } \\
\text { Einkommensunterschiede reduzieren }\end{array}$ & $30,9 \%$ & $40,3 \%$ & $+9,4$ \\
\hline darunter nach Altersgruppen: $14-25$ Jahre & $23,2 \%$ & $39,7 \%$ & $+16,5$ \\
\hline 25-55 Jahre & $32,1 \%$ & $40,4 \%$ & $+8,3$ \\
\hline $55+$ Jahre & $33,9 \%$ & $41,4 \%$ & $+7,5$ \\
\hline $\begin{array}{l}\text { Größtmögliche Zustimmung: Gleichbehandlung } \\
\text { und gleiche Chancen für alle }\end{array}$ & $32,7 \%$ & $37,6 \%$ & $+4,9$ \\
\hline
\end{tabular}

Hervorzuheben sind aus meiner Sicht die Abnahme des Vertrauens ins Rechtssystem, eine kritische Einstellung gegenüber der europäischen Integration, eine Abnahme des Vertrauens ins Europäische Parlament, der Wunsch nach Reduktion der Einkommensunterschiede (besonders in der Altersgruppe der 14bis 25-Jährigen) und eine Abnahme des Glücksempfindens. Hier zeigt sich einerseits, dass staatliche Institutionen, die rechtsstaatliche Prinzipien der Demokratie verkörpern, an Glaubwürdigkeit verloren haben, andererseits scheint auch die Idee einer staatenübergreifenden Gemeinschaft mit ihren Strukturen und Organisationen an Überzeugungskraft eingebüßt zu haben.

In Kapitel 8.2 kann man nachvollziehen, inwieweit die dargestellten Krisenfolgen und der Wandel des subjektiven Wohlbefindens in den Aussagen der interviewten Personen reflektiert werden. 


\title{
3.2 Exkurs: Auswirkungen des Börsenkrachs und der Weltwirtschaftskrise in den 1930er-Jahren, exemplarisch dargestellt an Zeitdokumenten der psychoanalytischen Community
}

\begin{abstract}
„Endlich kommt die merkwürdige Tatsache zur Wirkung, daß die Menschen im allgemeinen [sic!] ihre Gegenwart wie naiv erleben, ohne deren Inhalte würdigen zu können; sie müssen erst Distanz zu ihr gewinnen, d.h. die Gegenwart muß zur Vergangenheit geworden sein, wenn man aus ihr Anhaltspunkte zur Beurteilung des Zukünftigen gewinnen soll" (S. Freud, 1927c, S. 139).
\end{abstract}

In Anlehnung an dieses Zitat von Freud muss man in Betracht ziehen, dass Beurteilungen eines ökonomischen Ereignisses, überhaupt solche, die auf fundierten Analysen beruhen, den Menschen nicht unmittelbar zur Verfügung stehen. Auch wenn man dazu tendiert, im Rückblick die Geschichte als eine Folge von Ereignissen zu sehen, ist diese Betrachtungsweise nicht realitätsgerecht. Krisen ereignen sich in der Verzahnung von politischen, sozialen und ökonomischen Verhältnissen. So kann man die Weltwirtschaftskrise, die mit dem Börsenkrach 1929 in New York ihren Anfang nahm, auch nur in Zusammenhang mit den regionalen Gegebenheiten in Europa einer genaueren Analyse zuführen. Hier hatten die Menschen unter den Folgen des Ersten Weltkriegs zu leiden. Dass die Literatur manchmal als besserer Zeitzeuge fungiert, kann man an folgender Textpassage aus Stefan Zweigs „Die Welt von gestern“ erkennen, in der die Inflation bildhaft vor Augen geführt wird:

„Ein Nationalökonom, der all diese Phasen plastisch zu beschreiben wüsste, die Inflation in Österreich zuerst und dann in Deutschland, könnte nach meinem Gefühl an Spannung leicht jeden Roman übertreffen, denn das Chaos nahm immer phantastischere Formen an. Bald wusste niemand mehr, was etwas kostete. Die Preise sprangen willkürlich; eine Schachtel Zündhölzer kostete in einem Geschäft, das rechtzeitig den Preis aufgeschlagen hatte, das Zwanzigfache wie in dem anderen, wo ein biederer Mann arglos seine Ware noch zum Preise von gestern verkaufte [...] Bald kostete in Österreich eine mittelgroße Wohnung für das ganze Jahr ihren Mieter weniger als ein einziges Mittagessen; ganz Österreich hat eigentlich fünf oder zehn Jahre [...] mehr oder minder umsonst gewohnt. Durch dieses tolle Chaos wurde von Woche zu Woche die Situation widersinniger und unmoralischer. Wer vierzig Jahre gespart und überdies sein Geld patriotisch in Kriegsanleihe angelegt hatte, wurde zum Bettler. Wer Schulden besaß, war ihrer ledig. Wer korrekt sich an die Lebensmittelverteilung hielt, verhungerte; nur wer sie frech überschritt, aß sich satt. Wer zu bestechen wusste, kam vorwärts; wer spekulierte, profitierte [...] Es gab kein Maß, keinen Wert innerhalb dieses Zerfließens und Verdampfens des Geldes; es gab keine Tugend als die einzige: geschickt, geschmeidig, bedenkenlos zu 
sein und dem jagenden Ross auf den Rücken zu springen, statt sich von ihm zertrampeln zu lassen" (Zweig, 1987, S. 334 f.).

Wie die Reaktionen der Einzelwissenschaften und im gegebenen Fall der Psychoanalyse auf Krisen ausfallen, ist ein Hinweis darauf, auf welche Weise die gesellschaftspolitischen Verhältnisse Eingang in den theoretischen und klinischen Diskurs finden.

Bei Recherchen auf der Datenplattform PEP (Psychoanalytic Electronic Publishing) nach den Begriffen ,Arbeitslosigkeit“ $(17)^{35}$, „Wirtschaftskrise“ (2), „financial crisis“ (4), „financial losses“ (10), „loss of money“ (18), ,economic crisis“ (5), ,great depression“ (5), „bankruptcy“ (9), ,inflation“ (29), ,political crisis“ (2), „Black Friday“ (4), „Black Tuesday“ (0) zwischen 1928 und 1940 landet man nur wenige Treffer. Diese sind in Zahlen nach den Begriffen angeführt. Dazu kommt, dass die Begriffe Inflation und ,great depression“ mehrdeutig sind und, so gesehen, die Treffer noch einmal eingeschränkt werden. Gleiches gilt, wenn man in Betracht zieht, dass einige dieser Treffer, wie zum Beispiel „financial losses“ und „Arbeitslosigkeit“, auf individuelle Krankengeschichten bezogen sind. Hier stehen klinische Aspekte, wie der Zusammenhang zwischen Krisen und Suizidalität ${ }^{36}$ oder die Auswirkungen von Arbeitslosigkeit und Armut auf die Psyche der Betroffenen, also die „Psychologie des Geldes“ in Zusammenhang mit Verlusten, im Vordergrund.

35 Schmideberg (1935) fordert in ihrem Vortrag etwa, dass das Zusammenwirken von wirtschaftlichen und psychischen Faktoren genauer untersucht werden müsste, und zeigt am Beispiel der Arbeitslosigkeit auf, dass die Betroffenen nicht nur ihr Einkommen, sondern auch die Möglichkeit der Sublimierung ihrer Triebe verlieren. Die Folge ist ein regressiver Zustand, in dem die Menschen rationalen und irrationalen Ängsten preisgegeben sind. So werden äußere Ereignisse oft zu Projektionsflächen, an denen innere Konflikte ausgetragen werden. „Den meisten Menschen wäre es herzlich gleichgültig, ob es in einem entfernten Land $\mathrm{zu}$ einer Revolution kommt, und selbst die möglicherweise schlechte Finanzpolitik Amerikas würde ihnen nicht nahegehen, wäre es nicht wegen ihrer auf diese Interessen übertragenen unbewussten Konflikte“" (Schmideberg, 1935, S. 451).

36 Siehe dazu Lewis $(1933,1934)$ und Meng (1929). „Daß materielle Sorgen nicht selten den Impuls zum Selbstmord auslösen, zeigt die Statistik. In Zeiten der Arbeitslosigkeit und der Inflation und anderer wirtschaftlicher Krisen stieg die Selbstmordziffer. Die Konflikte, die ein Mensch erlebt, der arbeiten will, aber keine Arbeit findet, können unter bestimmten Voraussetzungen zum Selbstmord führen. Alles, was die materielle, körperliche und seelische Sicherheit eines Menschen bedroht, muß Vernichtungstendenzen gegen sich und andere aufwühlen. Käthe Kollwitz und Zille rufen immer wieder das Gewissen der materiell gesicherten Menschen wach, mitzuarbeiten an der Schaffung einer würdigen, sozialen Ordnung“ (Meng, 1929, S. 351). 
Eine Arbeit, die sich dezidiert mit der Wirtschaftskrise in Amerika auseinandersetzt, ist der Aufsatz von Wolfe „Psycho-analyzing the Depression“ (1932), der nicht auf der Datenplattform zu finden ist. Wolfe analysiert scharfsinnig die Krisenbewältigungsmechanismen der amerikanischen Bevölkerung, die sich in einem Spektrum von hedonistischen Ablenkungsszenarien, Verleugnung, masochistischen Selbstbeschuldigungen, Schuldzuweisungen an Sündenböcke, Flucht in die Vergangenheit - um nur einige zu nennen - bewegen, während er in der Übernahme von sozialer Verantwortung im Gegensatz zum gefeierten Individualismus, in besserer Bildung und Erziehung, die nicht auf autoritären Prinzipien beruht, und in Politisierung einen Ausweg sieht. Seine Beschreibungen der Abwehrmechanismen bei der Bewältigung der Krise sind durchaus aktuell, genauso wie die entzauberten amerikanischen Mythen von Erfolg und Leistung.

"Men who have never questioned the believe that hard work, honesty, and the investment of one's savings in, sound' stocks and bonds would eventually guarantee a comfortable security, are wearily pounding the streets in search of a job. Others who have retained their jobs have developed a panicky slave-morality toward their employers [...] The never-before-questioned rightness of unrestrained competitive individualism has proved itself a fatal boomerang. The great American myth has been exploded" (Wolfe, 1932, S. 209).

Die Ergebnisse der Kurzrecherche legen nahe, dass aus dem oben genannten Grund der fehlenden Distanz, aber womöglich auch wegen eines eingeschränkten Interesses vieler Psychoanalytiker für gesellschaftliche Belange, die über das Leiden der Individuen hinausgehen, die Treffer gering ausfallen, obwohl man aufgrund der gravierenden Auswirkungen der Weltwirtschaftskrise anderes erwarten würde.

Die geringe Publikationsdichte könnte aber auch der Tatsache geschuldet sein, dass Freud die Psychoanalyse aus der politischen Diskussion heraushalten wollte, in die man unweigerlich geraten wäre, hätte man die verheerende ökonomische Lage vieler Menschen nach dem Ersten Weltkrieg ins Auge gefasst und einer politischen Analyse unterzogen, wie es etwa Wilhelm Reich, der aktiv politisch tätig war, tat. ${ }^{37}$

Eine umfassende Darstellung der Reaktionen der psychoanalytischen Gemeinschaft auf den Börsenkrach und die Jahre der Depression in Europa und Übersee würde den Rahmen dieser Arbeit bei Weitem sprengen, wäre aber an sich von großem Interesse und lohnenswert, weil sie nicht nur über Arbeits- und Lebensbedingungen der Analytiker und Patienten, über spezifische Krankheitsbilder, sondern auch über das politische Engagement und seine Auswirkungen

37 Vergleiche dazu Kapitel 5.1.3 zu Reich. 
auf die institutionenspezifischen Bedingungen in den analytischen Vereinen Auskunft geben könnte.

Was im Rahmen dieser Arbeit geleistet werden kann, ist, einige der Rechercheergebnisse einzuarbeiten und exemplarisch nach Hinweisen auf die wirtschaftspolitische Situation in den Arbeiten Freuds zu suchen, die in den 1930erJahren veröffentlicht wurden, und sie durch Auszüge aus Briefen und Protokollen zu ergänzen. Am Beispiel der Geschichte des psychoanalytischen Verlags soll auf diese Weise ein Stück Institutionengeschichte vor dem Hintergrund der ökonomischen Entwicklung verdeutlicht werden. Hier wird sich zeigen, dass die wirtschaftliche Situation sehr wohl Einfluss auf Produktionsbedingungen genommen hat und in der unheilvollen Allianz mit dem Erstarken des Nationalsozialismus' im doppeldeutigen Sinn existenzbedrohend wurde.

Quinodoz weist darauf hin, dass Freud das Manuskript zu seinem Essay „Das Unbehagen in der Kultur“" wenige Tage nach dem Zusammenbruch der New Yorker Börse ablieferte. Er schreibt:

„Ein Jahr später, im September 1930, erzielte die Nazi-Partei bei den Reichstagswahlen einen gewaltigen Stimmenzuwachs, der Hitler den Weg zur Macht bahnte. Diese unheilvollen Ereignisse veranlassen Freud 1931, der 2. Auflage des Buches einen Satz anzufügen. Hatte er zuvor noch, was den Ausgang des Kampfes zwischen Eros und Todestrieb anbetrifft, eine gewisse Hoffnung erahnen lassen, fährt er nun fort: ,Aber wer kann den Erfolg und Ausgang voraussehen?'“ (Quinodoz, 2011, S. 390)

Diese Argumentation ist durchaus nachvollziehbar, wenn auch die Skepsis Freuds anders motiviert sein könnte. Letztlich hat die Geschichte die Frage, ob die Kultur fähig ist, die Aggression zu binden, Freuds skeptischer Position Recht gegeben. Die individuelle Geschichte ist untrennbar verknüpft mit der sozialen Realität, mit den politischen, ökonomischen und kulturellen Gegebenheiten.

Quinodoz macht in diesem Zusammenhang auf einen Brief Freuds an Arnold Zweig aufmerksam, der auf den 7. Dezember 1930 datiert ist. In diesem Schriftstück verleiht Freud seinen pessimistischen Zukunftsgedanken Ausdruck: „Wir leben schlechten Zeiten entgegen; ich sollte mich mit der Stumpfheit des Alters darüber hinwegsetzen, aber ich kann's nicht helfen, daß mir meine sieben Enkel leid tun“ (zitiert nach Quinodoz, 2011, S. 390).

Interessant ist in diesem Zusammenhang, dass dem Zitat ein Bedauern Freuds über das gescheiterte „Sowjetexperiment" vorausgeht, sodass ein direkter Bezug zur politischen Lage hergestellt werden kann. „Wir sind durch dasselbe [Sowjetexperiment] um eine Hoffnung - und eine Illusion - ärmer geworden und haben keinen Ersatz dafür" (E. L. Freud, 1980, S. 37).

Freud selbst hat, wie das folgende Zeugnis veranschaulicht, sich auch aktiv als Helfender eingebracht und seine Bekanntheit im Sinne einer Vorbildwirkung ins Treffen zu führen gewusst; andere sollten, so sein Wunsch, seinem Beispiel 
folgen. Drückende Not, Kälte, Hunger, Armut und Arbeitslosigkeit verschlimmerten die Lebensbedingungen der Menschen, besonders die von der Krise betroffenen Erwerbslosen, beträchtlich.

Im Jahr 1931 führte die Wiener Stadtverwaltung die sogenannte „Winterhilfe" ein, um diese Not ein wenig zu lindern. Im weiter unten zitierten Brief an Professor Julius Tandler, den Leiter des Wohlfahrtsamtes der Stadt Wien, erklärt sich Freud zu einer Art Selbstbesteuerung im Dienst der „Winterhilfe“ bereit und empfiehlt sein Beispiel, sollte es auf Zustimmung von Professor Tandler stoßen, der Nachahmung:

„Ich verpflichte mich, zum Beispiel von dem Erwerb jeden Tages, also täglich, mit Ausnahme der Sonntage und etwaiger Krankheitszeiten, den Betrag von S[chilling] 20 für die Zwecke der, Winterhilfe a abzugeben, solange diese Institution ihre Tätigkeit ausübt" (S. Freud, 1931g, S. 719).

Wie schwierig die Situation auch für die psychoanalytische Gemeinschaft war, ersieht man daran, dass der für 1931 geplante internationale psychoanalytische Kongress auf 1932 verschoben werden musste. In einem Brief vom 10. Jänner 1932 an Freud drückte Ernest Jones noch seine Zweifel aus, ob der Kongress überhaupt stattfinden würde: „Economically things are bad here, and I suppose they are no better on the Continent. I wonder if the Congress will be held this year. I hope so, also we must expect it to have a much smaller attendance than hitherto" (Paskauskas, 1993, S. 688).

Wiesbaden war der neue Konferenzort. Anna Freud berichtet im „Report of the Twelfth International Psycho-Analytic Congress":

"The President, Dr. Max Eitingon, opened the Congress on Sunday, September 4, at 9 a.m. at the Small Assembly Hall of the Kurhaus with the following address: I wish to thank all those present for having come to our Congress in spite of all the difficulties in their way and the distress and exigencies of the times. The Twelfth International Psycho-Analytical Congress, for which we have assembled here to-day, is taking place a year later and in another place than had been decided at our last meeting, held at Oxford in July, 1929. As you all know, the present Congress was to have taken place in Switzerland, at Interlaken, at the beginning of September, 1931. In July of last year the economic situation in Central Europe suddenly became vastly worse and nobody could then foresee how rapidly it might develop or what its effects would be. The Central Executive therefore thought it advisable, though it was very loth to take the decision, to put off the Congress for a year. Although the preparations were already well advanced, we postponed it to the beginning of September of this year, still intending that it should be held in Switzerland, at Interlaken. However, at the beginning of this year various local Societies requested the Central Executive, in view of the fact that everywhere the economic situation was growing steadily worse, to choose a 'cheaper' country for the Congress than beautiful Switzerland" (A. Freud, 1933, S. 138). 
Die Schwierigkeiten des Zusammenkommens sind offensichtlich, dennoch wird der Einfluss der ökonomischen und politischen Situation thematisch in den Vorträgen nicht aufgegriffen, sehr wohl jedoch in den Berichten von den einzelnen psychoanalytischen Instituten, die aufgrund der wirtschaftlichen Gegebenheiten zu kämpfen haben. Eitingon berichtet vom Berliner Institut:

"The economic crisis has made it very difficult for many of our colleagues to fulfil the condition according to which every member of the Society has to treat one case for the Institute free of charge. The economic situation has caused the resources of the Institute to be very seriously reduced and even jeopardized, for we have never had capital to secure our future. It is only through the formation of a little group of friends of the Berlin Psycho-Analytical Institute that we have been able to face the immediate danger, and with the support of these friends we hope to be able to carry the Institute on into better times" (A. Freud, 1933, S. 172).

Ähnliches lässt auch Helene Deutsch vom Wiener Institut hören:

"The number of students is steadily increasing, even though here as elsewhere the financial world-situation must inevitably make itself felt. It is rather remarkable, though explicable for us on the spot, that the candidates who have suffered most from the economic difficulties are those of our own country. Both in the medical and the philosophical schools of the University (the faculties of psychology and pedagogy), the young students of Vienna are becoming more and more eager to train in psycho-analysis. Many of the candidates are unable to bear the cost of the training themselves and formerly the Institute was able to help them with small scholarships. Now our resources have come to an end and the result is that the students at the Institute are for the most part recruited from abroad" (A. Freud, 1933, S. 175).

Die Aktivitäten des Wiener Instituts umfassen verschiedenste Technik- und Theorieseminare, besonders Pädagogen scheinen vom Institut angezogen zu sein, was Deutsch zu einer selbstkritischen, aber womöglich sehr weitsichtigen Bemerkung in ihrem Bericht veranlasst:

"As our syllabuses show, the social sciences have unfortunately come off very badly. The branches of work which I have described have absorbed so much time and interest that there was not enough left for these sciences" (A. Freud, 1933, S. 176).

An dieser Stelle fügt sich eine ironische Bemerkung Freuds (1937c) gut ein, in der er, in Anspielung ${ }^{38}$ auf den Börsenkrach, nicht nur den europäischen und amerikanischen Way of Life einander gegenüberstellt, sondern auch die genaue, langwierige klinische Arbeit den Wunschphantasien einer schnellen Heilung. Freud äußert sich am Beginn der Schrift kritisch gegenüber Bestrebungen, die

38 Siehe dazu Fußnote 1 (S. Freud, 1937c, S. 358). 
analytische Kur zu verkürzen, und bezieht sich an dieser Stelle ausdrücklich auf Rank, der mit der Beseitigung des Geburtstraumas die Quelle der Neurose trockengelegt zu haben glaubte.

„Der Versuch Ranks war übrigens aus der Zeit geboren, unter dem Eindruck des Gegensatzes von europäischem Nachkriegselend und amerikanischer ,prosperity" konzipiert und dazu bestimmt, das Tempo der analytischen Therapie der Hast des amerikanischen Lebens anzugleichen. Man hat nicht viel davon gehört, was die Ausführung des Rankschen Planes für Krankheitsfälle geleistet hat. Wahrscheinlich nicht mehr, als die Feuerwehr leisten würde, wenn sie im Falle eines Hausbrandes durch eine umgestürzte Petroleumlampe sich damit begnügte, die Lampe aus dem Zimmer zu entfernen, in dem der Brand entstanden war. Eine erhebliche Abkürzung der Löschaktion wäre allerdings auf diese Weise zu erreichen. Theorie und Praxis des Rankschen Versuchs gehören heute der Vergangenheit an - nicht anders als die amerikanische ,prosperity" selbst" (S. Freud, 1937c, S. 357 f.).

In diesem Zusammenhang ist von Bedeutung, dass Rank, der lange Zeit Leiter des psychoanalytischen Verlags war, die Wertschätzung Freuds besaß und den Großteil der für den Aufbau des Verlags nötigen Arbeit im Alleingang geleistet hatte (vgl. Marinelli, 2009, S. 57), 1926 jedoch die Stelle wegen der schwierigen Bedingungen als Verlagsleiter aufgab, um in Amerika mehr Geld zu verdienen, was sicher nicht der alleinige Grund war, Wien zu verlassen. In einem Brief an Brill schreibt Freud im Jahr 1924:

„Es ist der Dollar, der ihn verlockt. Aber es kommen noch andere Motive dazu. Wahrscheinlich die Erschütterung durch meine schwere Erkrankung und die daraus folgende Angst, wenn ich verschwinde, seine Existenz zu verlieren. Ferner die Verlockung, in der Analyse Entdeckungen zu machen, welcher alle unanalysierten Anfänger unterliegen. Es war ja bei Jung ebenso "(Lieberman, 2014, S. 321).

Marinelli hat in ihrem Buch „Psyches Kanon“ (Marinelli, 2009) die Entstehungsgeschichte des Internationalen Psychoanalytischen Verlags, der 1919 gegründet wurde, vor dem Hintergrund dieser prekären ökonomischen und politischen Situation nach dem Ersten Weltkrieg ausführlich beschrieben. Die Geschichte des Verlags kann als Beispiel dafür fungieren, wie ein Unternehmen in diesen ersten Nachkriegsjahren von nicht beeinflussbaren ökonomischen und politischen Gegebenheiten betroffen war.

„Diese Nachkriegsjahre der Inflation waren demgemäß auch die große Zeit der Spekulanten. Währungsspekulationen führten zu meist ebenso schnellen und glanzvollen Aufstiegen wie sie in den ökonomischen Abgrund führten. Das Banken- und Versicherungsgeschäft bevölkerten Glücksritter, deren aufwendiger Lebensstil von dem durch die Inflation und der neuen Umverteilungspolitik deklassierten Bürgertum mit Missfallen beobachtet wurde. So verlockend schien die Situation, dass auch 
Otto Rank einen Teil des Fonds-Geldes in dieses schnelle Geschäft investiert haben dürfte" (Marinelli, 2009, S. 49).

Die Finanzierung des Verlags stellte von Anfang an trotz des Verzichts Freuds auf sein Autorenhonorar und trotz der Spenden und Stiftungen ein ständiges Problem dar. Die Ressourcenknappheit im Energiebereich führte zu Papierengpässen und Vertriebsschwierigkeiten, und das Buch selbst wurde zu einem Spekulationsobjekt. Die Währungsinstabilitäten führten auch immer wieder zu Überlegungen, den Verlag von Wien nach Ungarn oder nach Berlin zu verlegen, was letztlich nicht geschah (vgl. Marinelli, 2009, S. 46 f.). Im Jahr 1929 verschärfte die Weltwirtschaftskrise die Situation des Verlages noch einmal, und erst eine Spendenaktion zu Freuds 75. Geburtstag im Jahr 1931 konnte den Bankrott abwenden. ${ }^{39}$

Dennoch hielten die finanziellen Schwierigkeiten an. Im Ringen um eine Lösung, den Verlag vor dem finanziellen Zusammenbruch zu bewahren, erfährt man aus dem Briefwechsel Freuds mit seinen Mitstreitern, wie es einzelnen von ihnen in dieser Zeit erging.

In einem Brief an Sándor Ferenczi liest man:

"You, too, will have heard in how sad a way the Eitingon firm has broken down, so that the members of the family are completely impoverished. Our friend has fallen ill with a paresis of the left arm, certainly under the effect of this material catastrophe" (S. Freud, 2000, S. 431).

Ernest Jones schreibt in einem Brief vom 21. April 1932 an Freud:

"It is not easy to be optimistic over any matter involving the raising of funds during the present terrible times. You know better than I do how bad things are in Austria, and now it is the turn of the English to be the poor cousins - since the currency relationship is the reverse of what it was ten years ago. Everyone here is having great

39 Marinelli schreibt dazu: „Getragen wurde diese Aktion von Analytikern und deren Patienten, die sich in eine unselige Verflechtung von finanziellen, therapeutischen und institutionellen Interessen begaben. Waren bis zu diesem Zeitpunkt die ärgsten Finanzkrisen mehr oder weniger hinter den Kulissen von Freud und Eitingon aus privater Tasche gelöst worden, so wurde spätestens mit dieser Sammelaktion die Finanzsituation des Verlags zur öffentlichen Angelegenheit erklärt" (Marinelli, 2009, S. 78).

Marinelli verweist auch auf die Anbindung des Verlags an die Internationale Psychoanalytische Vereinigung und die Herausgabe der „Neuen Folge der Vorlesungen zur Einführung in die Psychoanalyse" durch Freud zur Rettung des Verlags, weil sich auch die wirtschaftliche Situation seiner Förderer wie Max Eitingon, der einflussreicher Gesellschafter des Verlags war, im Zuge der Weltwirtschaftskrise massiv verschlechtert hatte (vgl. Marinelli, 2009, S. 81). 
difficulty in dealing with the immediate necessities of life. One is accustomed to look to America, but all that I hear from there is that the situation is terrible bad, with no immediate prospect of betterment, and in addition the economic depression has had a profounder psychological effect in producing apprehension there than in older countries better fortified to cope with misfortune" (Paskauskas, 1993, S. 692).

Dazu kam die Bedrohung durch die veränderte politische Situation mit der Erstarkung des Nationalsozialismus. Wieder geht es um den Verlag, wenn Freud an Jones am 12. September 1932 schreibt:

"Unfortunately the future of the Verlag depends on political developments in Germany; if this Hitlerei ${ }^{40}$ succeeds still further not many psa. books will be bought. The fate of my 'Ergänzende Vorlesungen', which have just gone to press, will be very enlightening" (Paskauskas, 1993, S. 709).

Mit der Machtergreifung der Nationalsozialisten, der Bücherverbrennung 1933, bei der auch Freuds Schriften verbrannt wurden, verzahnte sich die ökonomische Situation des Verlags mit der politischen auf eine bedrohliche Weise, die letztlich über die Beschlagnahmung und Verkaufsverbote der Bücher zur Auslöschung des Verlags und zur Einstellung seiner Publikationen führte. ${ }^{41}$ An dieser Stelle soll noch einmal Freud zu Wort kommen, der in der Antwort auf die Geburtstagwünsche von Jones vom 3. Mai am 7. Mai 1933 schreibt:

"You are right that, compared to my seventieth birthday, my worries are no longer focused on psychoanalysis. It is secure, and I know it to be in good hands. But the future of my children and my grandchildren is bleak and endangered, and my own impotence is painful" (Paskauskas, 1993, S. 719).

Interessanterweise hatte Jones in eben diesem Brief von einem Vorhaben berichtet, das nie ausgeführt wurde. "I am trying to write a book on $\mathrm{P} \alpha$ and World

40 Hervorhebung durch Freud.

41 Die Zeitschrift „Die Psychoanalytische Bewegung“ wurde 1933 eingestellt, die „Zeitschrift für psychoanalytische Pädagogik“ 1937. „Die Autoren mussten für ihre Veröffentlichungen die anfallenden Herstellungskosten selbst übernehmen. Auch der kleine Kreis der Gesellschafter des Verlags war weiter geschrumpft. Durch Eitingons Emigration nach Palästina und Ferenczis Tod bestand 1934 die Verlagsgesellschaft nur mehr aus Anna und Sigmund Freud“" (Marinelli, 2009, S. 83).

„1936 wurde dann zu jenem Jahr, welches das endgültige Aus für den deutschen Buchhandel bedeutete: in diesem Jahr begann die ,Arisierungsaktion' des Deutschen Buchhandels, in deren Zuge die ,jüdischen' Verlage aus dem Buchhandel verschwanden“ (Marinelli, 2009, S. 84). Wie Marinelli schreibt, erfolgte die ,juridische Löschung“" des Verlags 1941 (vgl. Marinelli, 2009, S. 88). 
Problems (government, politics, nationality, economics), and you may be sure it will have no "tendencies"” (Paskauskas, 1993, S. 718).

In Kapitel 5 werden neben Freud jene Psychoanalytiker zu Wort kommen, die in ihren Arbeiten die Wechselwirkung von sozioökonomischen Verhältnissen und psychischen Strukturen analysiert und dabei tendenziöses Grenzland betreten haben. Bernfelds Ausführungen zum „sozialen Ort" und Fromms Arbeiten zum „gesellschaftlichen Unbewussten“ sind bestimmende Faktoren in der Auswertung der Interviews.

Open Access Dieses Kapitel wird unter der Creative Commons Namensnennung 4.0 International Lizenz (http://creativecommons.org/licenses/by/4.0/deed.de) veröffentlicht, welche die Nutzung, Vervielfältigung, Bearbeitung, Verbreitung und Wiedergabe in jeglichem Medium und Format erlaubt, sofern Sie den/die ursprünglichen Autor(en) und die Quelle ordnungsgemäß nennen, einen Link zur Creative Commons Lizenz beifügen und angeben, ob Änderungen vorgenommen wurden.

Die in diesem Kapitel enthaltenen Bilder und sonstiges Drittmaterial unterliegen ebenfalls der genannten Creative Commons Lizenz, sofern sich aus der Abbildungslegende nichts anderes ergibt. Sofern das betreffende Material nicht unter der genannten Creative Commons Lizenz steht und die betreffende Handlung nicht nach gesetzlichen Vorschriften erlaubt ist, ist für die oben aufgeführten Weiterverwendungen des Materials die Einwilligung des jeweiligen Rechteinhabers einzuholen.

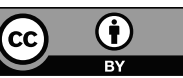

